\title{
Broadband Cavity Enhanced Absorption Spectroscopy: A New Approach to Search for DIB Carriers
}

\author{
A. J. Walsh ${ }^{1}$, D. Zhao ${ }^{1}$, W. Ubachs ${ }^{2}$ and H. Linnartz ${ }^{1}$ \\ ${ }^{1}$ Raymond and Beverly Sackler Laboratory for Astrophysics, Leiden Observatory, \\ University of Leiden, P.O. Box 9513, NL 2300 RA Leiden, The Netherlands. \\ email: linnartz@strw.leidenuniv.nl \\ ${ }^{2}$ LaserLaB, Department of Physics and Astronomy, VU University, \\ De Boelelaan 1081, NL 1081 HV Amsterdam, The Netherlands
}

\begin{abstract}
A new and sensitive set-up to swiftly test proposed carriers of the diffuse interstellar bands (DIBs), over a relatively broad spectral range, is described. The instrument utilizes broadband cavity enhanced absorption spectroscopy (BBCEAS) and incorporates an optomechanical shutter to modulate light from a continuous incoherent light source. A pulsed supersonically expanding planar plasma expansion is used to mimic conditions in translucent interstellar clouds. Measurements of plasma durations as low as $400 \mu$ s are possible. The sensitivity is estimated to be better than $10 \mathrm{ppm} /$ pass, measured with an effective exposure time of only ca. $1 \mathrm{~s}$. The performance and potential of the instrument is demonstrated on spectra of $\mathrm{C}_{5} \mathrm{H}, \mathrm{C}_{6} \mathrm{H}$ and $\mathrm{C}_{9} \mathrm{H}_{3}$ recorded through expanding hydrocarbon plasma.
\end{abstract}

Keywords. Laboratory astrophysics, spectroscopy, supersonic plasma jet

\section{Introduction}

Several families of DIB carrier candidates have been proposed and investigated in the laboratory, including PAH cations (e.g. Salama et al. 2011), carbon chain radicals (e.g. Nagarajan \& Maier 2010), fullerenes (e.g. Foing \& Ehrenfreund 1994), and excited molecular hydrogen (e.g. Sorokin and Glownia 1996), yet still no definite match between laboratory and astronomical data could be made. Testing these candidates has presented a large experimental challenge, simulating the cold harsh conditions of the interstellar medium under fully controlled conditions, and a number of different techniques have been used. Spectroscopic surveys using mass selective low-temperature matrix isolation tools (e.g. Nagarajan \& Maier 2010) can cover a large wavelength domain in a short time and are a useful guide for where to search for the unperturbed gas phase spectra. Molecular beams and jets coupled with discharge (e.g. Motylewski \& Linnartz 1999) or laser vaporization/photolysis sources (e.g. Giesen et al. 1994) have been used to generate adiabatically cooled transients, as in the diffuse interstellar medium intense radiation fields are expected to favour formation of unstable species. REMPI-TOF, electron detachment, plasma-frequency double modulation, ion trap and degenerate four-wave mixing are among the techniques that have been used to search with high sensitivity and high precision for accurate gas phase spectra of possible DIB carriers, but typically their spectral scanning speed is slow. All relevant references are summarized in Walsh et al. (2013). Cavity enhanced technology offers an alternative and is the topic of this contribution.

High sensitivity can be realised using cavity enhanced absorption spectroscopy (CEAS) techniques (see Berden \& Engeln 2009 for review). Consequently, cavity ring-down 
spectroscopy has been frequently applied for measurements of carrier candidates (Motylewski et al. 2000). In a conventional CRD scheme, light from a pulsed laser is incident on an optically stable cavity, containing the sample under investigation, constructed by two high reflectivity (HR, typically $R>99.99 \%$ ) plano-concave mirrors. A small portion of light enters the cavity and is trapped between the two HR mirrors. Subsequently, the absorbance of the sample can be retrieved by fitting a mono-exponential decay to the time dependent intensity of light leaking out of the cavity, referred to as the ring-down time, resulting in absorbance sensitivity better than $10^{-6}$ per pass. A practical drawback of conventional CRD is that only a single wavelength absorption can be measured from a single ring-down event, a stepwise approach is necessary to record a spectrum. This approach is generally very time-consuming when covering large spectral ranges, and consequently not optimum when testing DIB carrier candidates. An alternative is offered by using a white light source instead of a laser. In recent yeears, a number of so called broadband CEAS (BBCEAS) techniques have been pioneered and applied to a variety of fields (Berden \& Engeln 2009), due to their broad spectral coverage, easy data acquisition and experimental simplicity. These properties are here adapted to search for DIB carriers.

In the present work, we introduce a new experimental concept, presenting a sensitive instrument capable of swiftly recording gas phase absorption spectra of transients created in a slit pulsed discharge nozzle (Walsh et al. 2013), with spectral resolution equivalent to astronomical observations, by employing BBCEAS (Fiedler et al. 2003). This facilitates proficient testing of potential candidates of DIB carriers. The performance of the technique is demonstrated on the basis of electronic transitions of a series of hydrocarbon species, a proposed carrier candidate as a number of carbon chains, bare and hydrogen/nitrogen/oxygen-capped, and related ions have been identified in dense interstellar clouds. The instrument is described from three aspects; production of carbon chain species using a pulsed discharge nozzle, observation of species using the BBCEAS setup, and the timing aspects of the instrument, combining a pulsed production source with a continuous detection technique.

\section{Experimental}

Production; High-pressure pulsed planar plasma expansions, have proved to be very successful in producing large abundances of highly reactive molecular transients, such as carbon chain radicals and polycyclic aromatic hydrocarbon cations (PAHs), at low rovibrational temperatures in an almost Doppler free environment. In the present set-up, a pulsed planar discharge expansion of $0.5 \% \mathrm{C}_{2} \mathrm{H}_{2}$, diluted in a 1:1 $\mathrm{He} / \mathrm{Ar}$ precursor gas mixture is employed to form carbon chain transients. The production technique has been described in detail elsewhere (Motylewski \& Linnartz 1999) and will only be discussed briefly here, with typical settings for the measurements presented. A supersonically expanding gas pulse of $2 \mathrm{~ms}$ is released into a vacuum chamber. The dispersed gas pulse travels through a grounded metallic plate, with a slit, and two metal jaws. The two metal jaws act as cathodes. The grounded plate and cathode jaws are separated by a ceramic spacer, also with a slit. The ground plate is separated from the nozzle body by a second slotted ceramic spacer. A negative voltage of approximately $1200 \mathrm{~V}$ is applied to the metal jaws for between 500 and $1000 \mu \mathrm{s}$, as the expanding gas pulse moves through the nozzle system and strikes toward the grounded metal plate. The gas pulse is monitored by measuring the voltage drop, and current increase, across one of the jaws of the slit (Figs. 1(a) and (b)).

Detection; BBCEAS is employed for detection. In BBCEAS, the wavelength dependent integrated transmission of light from a continuous light source through a high finesse 

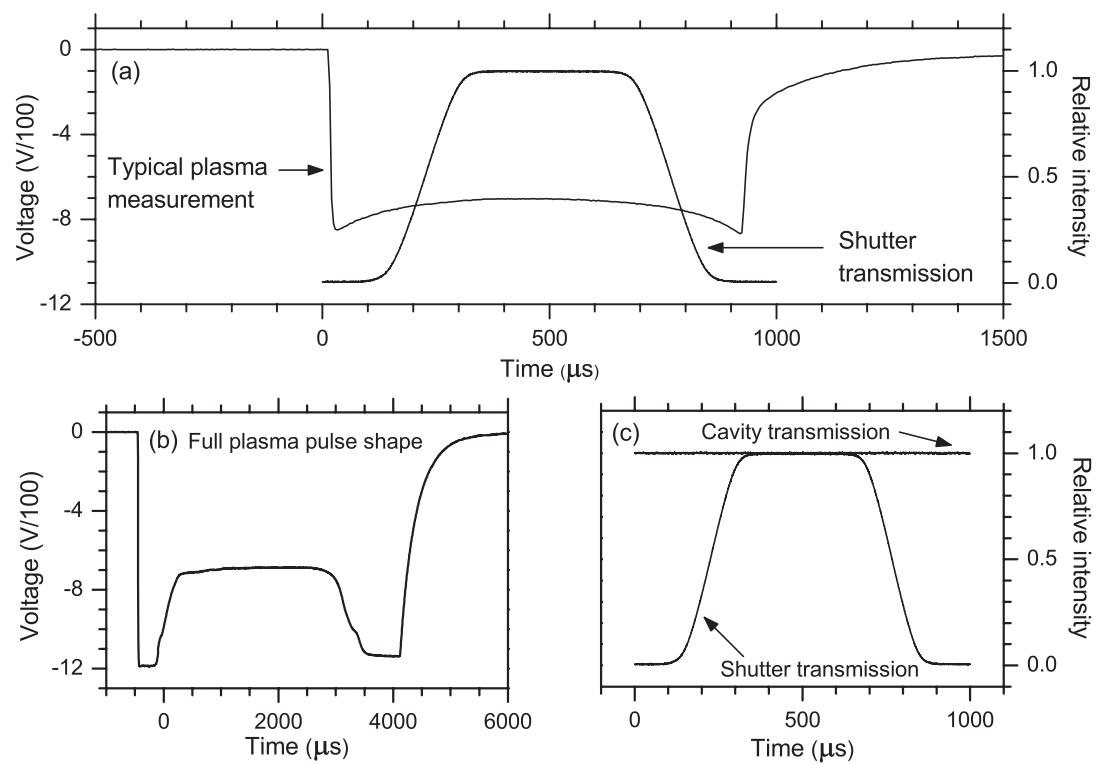

Figure 1. Light exiting the cavity is only transmitted through the shutter when the plasma strikes and is monitored using a photodiode. The signal from the photodiode and voltage at one of the jaws is made to coincide in time using a set of pulse and delay generators. Typical overlapping signals from the photodiode and plasma are shown in (a). The temporal profile of the full gas pulse, through the slit, is visualized by extending the length of the discharge pulse to several milliseconds and by monitoring the voltage at one of the jaws; see (b). Comparison of the relative intensity of light exiting the cavity with light transmitted through the shutter, measured using a photodiode is shown in (c).

cavity is measured, with the sample to be investigated $(I)$ and without the sample $\left(I_{0}\right)$. The absorbance can be calculated using $A=\left(I_{0} / I-1\right)(1-R)$, where $R$ is the reflectivity of the mirrors. In the present setup, a pulsed discharge nozzle is aligned with its slit along and approximately $7 \mathrm{~mm}$ above the optical axis of a high finesse cavity. The optical cavity comprises two identical HR mirrors, $\approx 99.995 \%$ reflectivity between 520 and $560 \mathrm{~nm}$. Light from a $300 \mathrm{~W}$ Xe-arc lamp is coupled into the optical cavity using a small telescope. Light leaking out of the cavity is focused onto the entrance of a homemade optomechanical shutter, which only transmits light when the plasma is present. Light transmitted through the optomechanical shutter is incident on a beam splitter, which diverts $10 \%$ of the light onto a photodiode, see below. The broad-band light transmitted through the beam splitter is spectrally narrowed by an interference bandpass filter to the spectral range of the HR mirrors and is focused onto a circular to rectangular optic fiber bundle. Light exiting the optical fiber is incident on a $13 \mu \mathrm{m}$ slit entrance of a spectrograph. The spectrograph disperses light onto a charge-coupled device. The spectrometer can cover $20 \mathrm{~nm}$ at a time with a resolutipn of $0.03 \mathrm{~nm}$, which is sufficient to resolve the majority of the observed DIBs.

Timing; In a pulsed discharge nozzle source the sample exists for $500 \mu$ s and is generally employed at less than $20 \mathrm{~Hz}$, leading to an exceedingly low duty cycle (0.01); only $1 \%$ of light leaking out of the cavity is present coincidentally with the sample. This reduces signal-to-noise ratio $(\mathrm{S} / \mathrm{N})$ substantially, consequently also reducing sensitivity. To overcome this, we incorporate an optomechanical shutter as a time-gate, modulating the detection scheme of BBCEAS, in which way the effective duty cycle is improved and reaches a value close to unity. The shutter transmits light only when the plasma is present 


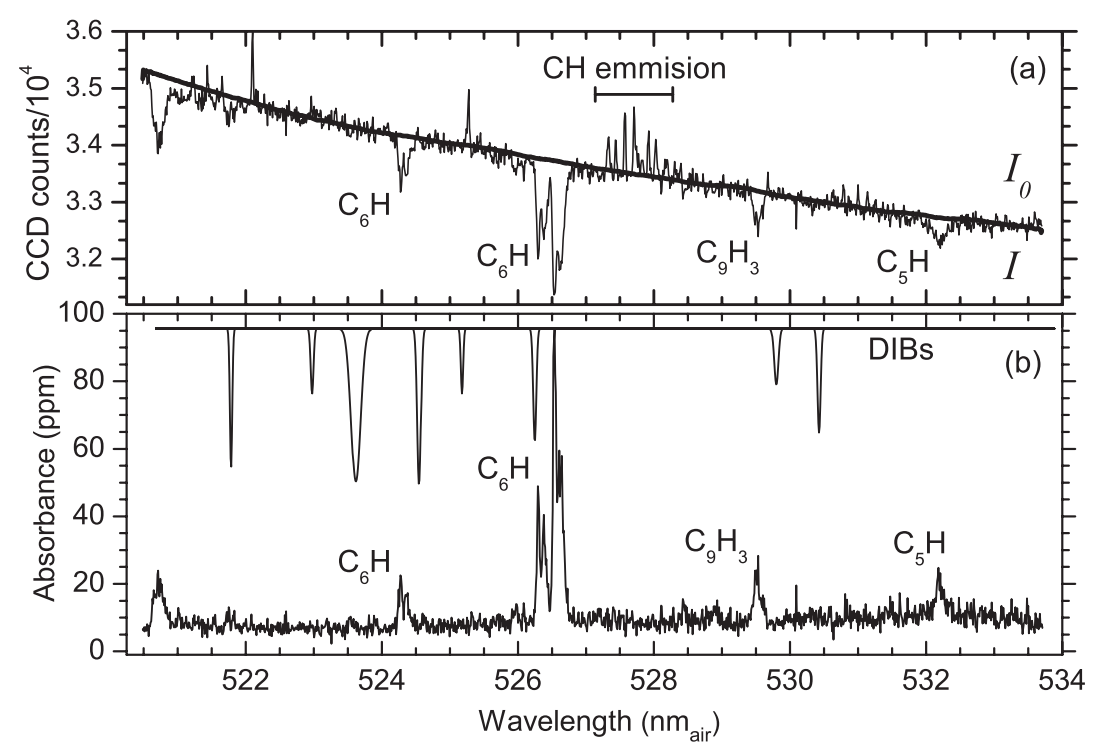

Figure 2. Sample $I_{0}$ and $I$ spectra, in absolute counts are shown in (a). $I_{0}$ is featureless and smoothened for analysis, shown in a thick black line. Processed spectrum of hydrocarbon plasma, in units of ppm per pass is shown in (b). DIBs observed in the spectrum of HD 204827 (Hobbs et al. 2008) are also shown. No coincidence between the measured absorption spectra and the DIBs occurs, however it is clear that DIBs can be directly compared with the present setup.

in the cavity. To monitor the opening/closing of the shutter, a beam splitter directs a portion $(10 \%)$ of the light transmitted through the cavity onto a photodiode. A comparison between the intensity of light leaking out of the cavity and light transmitted through the optomechanical shutter is shown in Fig. 1(c). The width of the discharge pulse is chosen to be slightly longer than the opening time of the shutter. The plasma and shutter are set coincidental using a set of pulse generators and delays. A sample signal of the overlapping voltage drop across one of the jaws of the discharge and the intensity of light transmitted through the shutter is shown in Fig. 1(a). Optomechanical shutter modulation is a necessity for the observation of weak transient absorptions in the plasma, improving the $\mathrm{S} / \mathrm{N}$ by a factor of 8 , compared to fast-time-gating of a linear array of pixels.

\section{Sample measurement}

In a standard measurement, the CCD is exposed to light for $90 \mathrm{~s}$ at a time, and data is accumulated for $15 \mathrm{~min}$. This results in an effective exposure time of only one second, for a plasma duration of $500 \mu \mathrm{s}$. A sample measurement of $I$ and $I_{0}$, between 520.5 and $534 \mathrm{~nm}$ is shown in Fig. 2(a). All values are given in nanometers in air. The $I_{0}$ spectrum is featureless, and is smoothened for analysis. Absorption features (sharp decreases in transmission) and emission features (sharp increases in transmission) can both be seen in the spectrum $I$. Emission of $\mathrm{CH}$ is clearly visible. Absorption features of $\mathrm{C}_{6} \mathrm{H}(524.5$, 526.5 , and $526.7 \mathrm{~nm}), \mathrm{C}_{9} \mathrm{H}_{3}(529.6 \mathrm{~nm})$, and $\mathrm{C}_{5} \mathrm{H}(532.4 \mathrm{~nm})$ are visible (Nagarajan \& Maier 2010). As the plasma itself is a light source, two background measurements are made: a background dark current, where the light source is blocked and the plasma is not running $(B G)$ and a background measurement while the plasma is running and the light source is blocked $\left(B G_{\text {plasma }}\right) . B G_{\text {plasma }}$ is subtracted from $I$, to remove the effects of light emission from the plasma. Reflectivity $(R)$ of the cavity mirrors in the present experiment are measured in a regular cavity ring down experiment in advance. 
The processed absorbance spectrum is shown in Fig. 2(b). In this figure tabulated DIBs are also shown to allow for comparison. It should be realized that the laboratory spectrum has been recorded during an effective exposure time of $1 \mathrm{~s}$, whereas for example a high resolution spectrum of the origin band of $\mathrm{C}_{6} \mathrm{H}$ at $\sim 526.7 \mathrm{~nm}$ measured using CRD takes nearly an hour. Using calculated oscillator strengths (Zhao et al. 2011), the $\mathrm{C}_{6} \mathrm{H}$ expansion densities are estimated to be $10^{10}-10^{11}$ molecules $/ \mathrm{cm}^{3}$. The maximum absorbance of the $\mathrm{C}_{6} \mathrm{H}$ R-branch peak of the $\mathrm{B}^{2} \Pi_{3 / 2}-\mathrm{X}^{2} \Pi_{3 / 2}$ spin orbit component is estimated from previous CRDS measurements to be $150 \pm 20$ ppm per pass (Zhao et al. 2011), in agreement with the absorbance measured here. The estimated sensitivity is better than $10 \mathrm{ppm}$, comparable to high resolution techniques and as the DIBs are generally broad, the somewhat lower resolution is not a real disadvantage at this stage.

\section{Conclusion}

In conclusion, we have introduced a new technique fully optimized for measuring absorptions of pulsed plasma events over a relatively large wavelength range, suitable for testing DIB carrier candidates, as the set-up offers a fast method to search for possible DIB matches with a sensitivity better than $10 \mathrm{ppm}$, comparable to other cavity enhanced techniques. It has a much larger spectral coverage than laser based gas phase experiments; in a single observation, features can be observed over a $20 \mathrm{~nm}$ range - determined by the wavelength selection devise and reflectivity curve of the mirrors - and with a resolution high enough to resolve the majority of the DIBs. Moreover, different signals of the same carrier molecule simultaneously can be observed, which allows absolute intensity comparisons, e.g., in a vibronic progression. In a similar way multiple species can be studied at once. Little data analysis is needed, and if necessary, portions of the spectrum can be complimented with further investigation by regular high resolution CRD. As the Xe-arc light source has a large spectral coverage, the setup also offers the prospect of examining carrier candidates up to the near-IR, a region where dye lasers, for example, are harder to operate.

The concept presented here is principally different from all previous techniques used to search for potential DIB carriers, and its potential lies particularly in its applicability. A full report is available from Walsh et al. (2013).

\section{References}

Berden, G. \& Engeln, R. (eds.) 2009, Cavity Ringdown Spectroscopy - Techniques and Applications (Wiley and Blackwell: Chester, United Kingdom)

Fiedler, S. E., Hese, A., \& Ruth, A. A. 2003, Chem. Phys. Lett., 371, 284

Foing, B. H. \& Ehrenfreund, P. 1994, Nature, 319, L59

Giesen, T., van Orden, A., Hwang, H., Fellers, R., Provencal, R., \& Saykally, R. 1994, Science, 265,756

Hobbs, L. M., York, D. G., Snow, T. P., Oka, T., Thorbum, J. A., Bishop, M., Friedman, S. D., McCall, B. J., Rachford, B., Sonnentrucker, P., et al. 2008, Astrophys. J., 680, 1256

Motylewski, T. \& Linnartz, H. 1999, Rev. Sci. Instrumen., 70, 1305

Motylewski, T., Linnartz, H., Vaizert, O., Maier, J. P., Galazutdinov, G. A., Musaev, F. A., Krelowski, J., Walker, G. A. H., \& Bohlender, D. A. 2000, Astrophys. J., 531, 312

Nagarajan, R. \& Maier, J. P. 2010, Int. Rev. Phys. Chem., 29, 521

Salama, F., Galazutdinov, G. A., Krelowski, J., Biennier, L., Beletsky, Y., \& Song In-Ok. 2011, Astrophys. J., 728, 154

Sorokin, P. P. \& Glownia, J. H. 1996, Astrophys. J., 473, 900

Walsh, A. J., Zhao, D., Ubachs, W., \& Linnartz, H. 2013, J. Phys. Chem. A, 117, 9363

Zhao, D., Haddad, M. A., Linnartz, H., \& Ubachs, W. 2011, J. Chem. Phys., 135, 044201 